Origin and simulation of sparks in MPGD

This article has been downloaded from IOPscience. Please scroll down to see the full text article.

2012 JINST 7 C06009

(http://iopscience.iop.org/1748-0221/7/06/C06009)

View the table of contents for this issue, or go to the journal homepage for more

Download details:

IP Address: 137.138.125.164

The article was downloaded on 09/07/2012 at 13:50

Please note that terms and conditions apply. 


\title{
Origin and simulation of sparks in MPGD
}

\author{
S. Procureur,,${ }^{a, 1}$ S. Aune, ${ }^{b}$ J. Ball, ${ }^{a}$ G. Charles, ${ }^{a}$ B. Moreno,,${ }^{a}$ H. Moutarde ${ }^{a}$ and \\ F. Sabatié ${ }^{a}$ \\ ${ }^{a}$ CEA, Centre de Saclay, Irfu/SPhN, \\ 91191 Gif sur Yvette, France \\ ${ }^{b}$ CEA, Centre de Saclay, Irfu/Sédi, \\ 91191 Gif sur Yvette, France \\ E-mail: Sebastien.Procureur@cea.fr
}

ABSTRACT: The development of Micro-Pattern Gaseous Detectors for high luminosity experiments requires a better understanding of the origin of the sparks in these detectors. Assuming a spark occurs whenever the electron number reaches the well known Raether limit, previous Geant4 simulations quantitatively reproduced the spark rate observed in Micromegas with high energy hadron beams. Large release of energies are provided by fragments from nuclear interactions between the beam and the detector material. In order to further check the validity of our simulation, hadron beam tests have been performed at the CERN/SPS and PS on Micromegas and hybrid Micromegas-GEM detectors. In particular, large variations of the spark rate have been observed in positively charged hadron beams below $1 \mathrm{GeV} / \mathrm{c}$, which are well described by the simulation. The role of the charge density has also been investigated with measurements in magnetic fields and with a Micromegas-GEM. The simulation has therefore been upgraded to take into account the transverse diffusion and is now able to quantitatively explain the role of a GEM foil in the spark rate reduction.

KEYWORDS: Gaseous detectors; Detector modelling and simulations II (electric fields, charge transport, multiplication and induction, pulse formation, electron emission, etc); Electron multipliers (gas); Particle tracking detectors (Gaseous detectors)

\footnotetext{
${ }^{1}$ Corresponding author.
} 


\section{Contents}

1 Introduction to the simulation of sparks 1

1.1 Geant4 and origin of sparks 1

1.2 Open questions 2

2 Sparks and beam energy 2

2.1 Tests at the CERN/SPS and PS 2

2.2 Results and simulation 3

2.2.1 High energy beams - SPS 3

2.2.2 Low energy beams - PS 4

3 Sparks and magnetic field $\quad 5$

3.1 Case of a transverse magnetic field 6

$\begin{array}{ll}3.2 & \text { Case of a longitudinal magnetic field }\end{array}$

4 Effect of the GEM foil on the spark rate $\quad 8$

4.1 Experimental observations 8

$\begin{array}{ll}4.2 & \text { Critical surface charge density }\end{array}$

$\begin{array}{lll}4.3 & \text { Comparison with data } & 9\end{array}$

5 Conclusion $\quad 10$

\section{Introduction to the simulation of sparks}

\subsection{Geant4 and origin of sparks}

Micro-Pattern Gaseous Detectors (MPGDs) are extensively used in several high energy experiments where very high fluxes require a fast collection of the signal $(\sim 100 \mathrm{~ns})$. In these experiments, the main limitation of the MPGDs comes from the development of sparks in the amplification region, which causes dead time and possible long term damage. Even if all the mechanisms leading to the formation of a spark are not precisely understood, the well known Raether limit states that a spark is likely to occur whenever the charge number exceeds a few $10^{7}$ [1]. This limit cannot be reached in standard conditions, i.e. in the detection of Minimum Ionizing Particles (MIPs) with gains below 10,000. Such gains require the release of thousands of primary electrons, which can only occur in catastrophic events where a highly ionizing particle (HIP) is produced in the vicinity of the detector. To confirm this interpretation, a Geant4 [2] based simulation of Micromegas [3] has been developed to study the production rate of HIPs [4]. It has been found that such particles mainly correspond to nuclear fragments from the interaction of an incident hadron and the detector material. Using a sparking condition a la Raether, a spark probability was derived and found to be 
in quantitative agreement with few measurements [5] performed in $15 \mathrm{GeV} / \mathrm{c}$ pion beams. Furthermore, the simulated spark probability exhibited the same power law dependence with the detector gain as in the data.

\subsection{Open questions}

Though the simulation gives a correct estimate of the spark probability for Micromegas in a $15 \mathrm{GeV} / \mathrm{c}$ beam, further studies and comparisons are needed to extend the validity of this model. The main goal of this extension is to provide a tool that could reliably predict the spark rate for a given setup. In particular, the effect of the following parameters should be investigated:

- The beam energy: the cross sections for the production of nuclear fragments are indeed energy dependent. Besides, the Geant 4 physics lists are not supposed to be equally valid at different energies.

- The detector thickness: thicker materials could enhance the production of HIPs. This is particularly an issue for the micro-mesh, with the recent development of the bulk technology [6].

- The presence of an external magnetic field: such a field modifies the drift of electrons and can therefore affect the local charge density in the gas. The case of a parallel and perpendicular magnetic field (with respect to the electric field) should be distinguished.

- The presence of a GEM [7] foil: it is well known that GEM detectors are less sensitive to the development of sparks. It has also been checked that the use of a GEM foil in combination with a Micromegas detector significantly reduces the spark rate [8]. A quantitative understanding of the underlying mechanism is still missing.

The study of all these parameters requires new measurements that could be compared with the simulation. In the last two years, three different beam tests were therefore organized both at CERN and at the Jefferson Laboratory to cover all the configurations.

\section{Sparks and beam energy}

\subsection{Tests at the CERN/SPS and PS}

The remarkable variety of beam lines available at CERN makes it a unique place to test the dependence of the spark probability on the beam energy. High intensity hadron beams can be used from $150 \mathrm{GeV} / \mathrm{c}$ (SPS) down to several hundreds of MeV/c (PS). A first campaign was organized in October 2009 on the SPS/H4 beam line, with the aim of measuring the spark probability for different types of Micromegas [9]. Six detectors could be placed simultaneously on a support structure to compare their behaviour in the same conditions. The whole structure was located inside the Goliath dipole to investigate the role of a transverse magnetic field.

The second beam test took place on the PS/T11 line in August 2010 [10], with twelve detectors tested simultaneously. The T11 beam line offers the possibility to tune the beam energy from $3 \mathrm{GeV} / \mathrm{c}$ down to approximately $200 \mathrm{MeV} / \mathrm{c}$, by changing currents in the upstream magnets. The charge of the particles can also be chosen, by reversing the sign of these currents. Negatively 
Table 1. CERN beam test specificities.

\begin{tabular}{|c|c|c|}
\hline & CERN/SPS & CERN/PS \\
\hline Beam line & $\mathrm{H} 4$ & $\mathrm{~T} 11$ \\
\hline Beam momentum & $150 \mathrm{GeV} / \mathrm{c}$ & $0.2-3 \mathrm{GeV} / \mathrm{c}$ \\
\hline Beam particles & $\pi$ & $\pi^{-}$or $\pi^{+}$with protons \\
\hline Spill & $10 \mathrm{~s}$ every $50 \mathrm{~s}$ & 1 or $2 \times 0.4 \mathrm{~s}$ every $50 \mathrm{~s}$ \\
\hline Max beam intensity & $10^{6} /$ spill & $5 \times 10^{5} /$ spill \\
\hline Number of detectors tested & 10 & 20 \\
\hline Active area of detectors & $10 \times 10 \mathrm{~cm}^{2}$ & $10 \times 5 \mathrm{~cm}^{2}$ \\
\hline Gas mixture & $\mathrm{Ar}+5 \% \mathrm{iC}_{4} \mathrm{H}_{10}$ & $\mathrm{Ar}+5 \% \mathrm{iC}_{4} \mathrm{H}_{10}$ \\
\hline Number of spills recorded & 7,000 & 27,000 \\
\hline
\end{tabular}
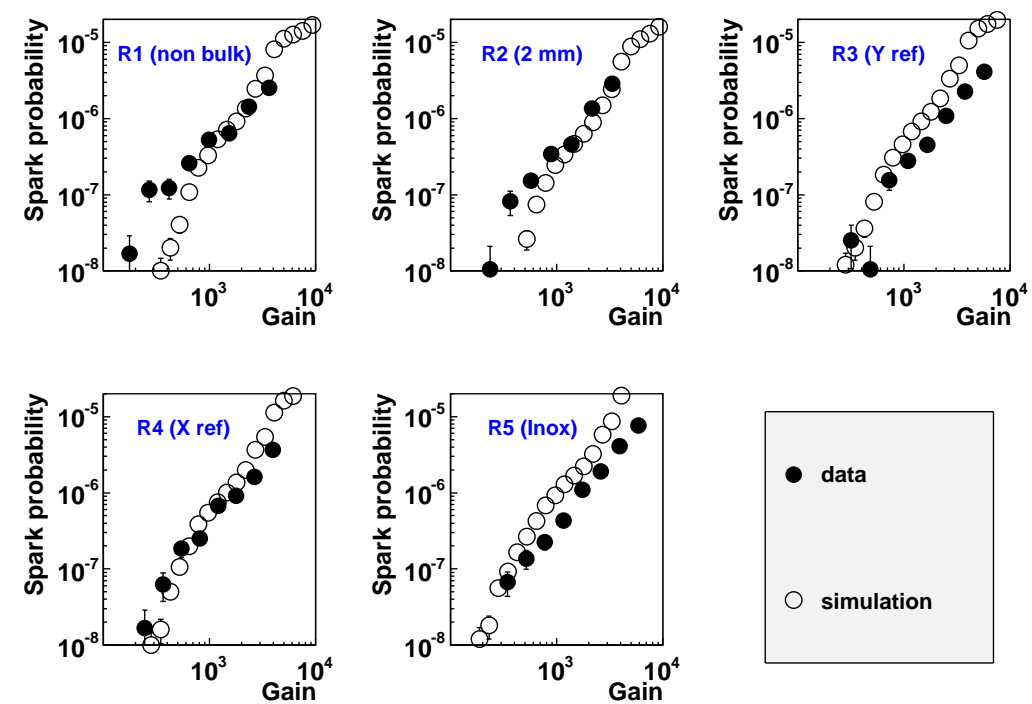

Figure 1. Discharge probability in a $150 \mathrm{GeV} / \mathrm{c}$ pion beam as a function of the gain for five Micromegas detectors. Measurements (full circles) are compared with the Geant4 simulation (open circles) [9]. R1 is a non bulk detector, $\mathrm{R} 2$ has a reduced, $2 \mathrm{~mm}$ drift gap, $\mathrm{R} 3$ and $\mathrm{R} 4$ are reference detectors, and $\mathrm{R} 5$ is equipped with a micro-mesh as the drift electrode.

charged beams essentially consist of $\pi^{-}$, with negligible contribution of anti-protons. In the case of positively charged particles however, the fraction of protons in the $\pi^{+}$beams reaches almost $1 \%$.

The main characteristics of these two beam tests are summarized in table 1 .

\subsection{Results and simulation}

\subsubsection{High energy beams - SPS}

The measured spark probability for five detectors is shown on figure 1, with a usual power law dependence as a function of the gain. In particular, no significant difference has been observed between thick and thin micro-mesh, i.e. between bulk and non bulk detectors. These results have 


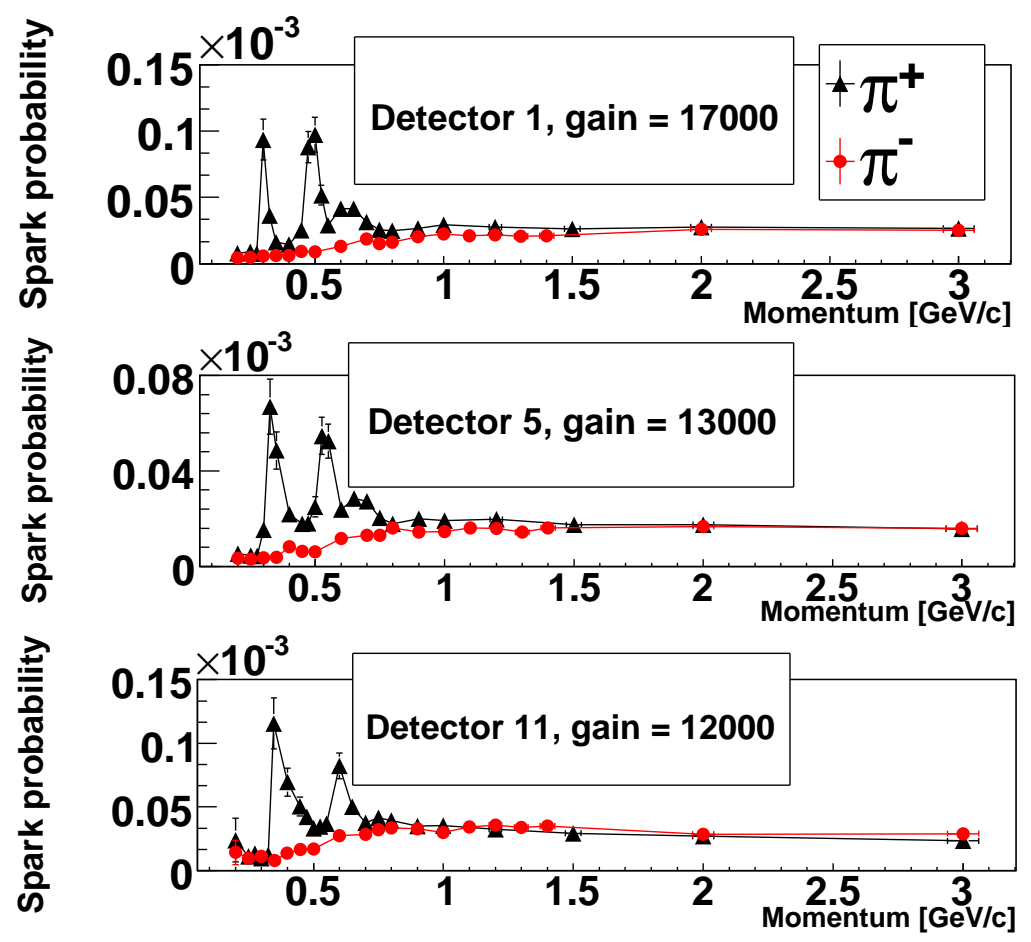

Figure 2. Discharge probability for three Micromegas detectors as a function of the beam energy in $\pi^{-}$and $\pi^{+}+$proton beams. The micro-mesh high voltage of all detectors was fixed at $430 \mathrm{~V} \mathrm{[10]}$.

been compared with a full Geant 4 simulation of the experimental setup, using the same physics list and the same method as in [4]. The only free parameter, i.e. the value of the Raether limit $N_{R}$, has been adjusted to the data. A reasonable agreement is obtained with $N_{R}=2.5 \times 10^{7}$, which is close to the value fitted from $15 \mathrm{GeV} / \mathrm{c}$ data, $2 \times 10^{7}$.

\subsubsection{Low energy beams - PS}

At low energy, scans in both gain and beam energy have been performed. The latter is illustrated in figure 2 for three detectors located at the upstream, middle and downstream part of the setup. Above $1 \mathrm{GeV} / \mathrm{c}$, results for negatively and positively charged beams are well compatible, and almost energy independent. Below $1 \mathrm{GeV} / \mathrm{c}$ however, a significant decrease is observed in the $\pi^{-}$case, while three pronounced peaks appear at specific momenta of the $\pi^{+}$and protons, namely 300,500 , and $650 \mathrm{MeV} / \mathrm{c}$. The latter one is wider and almost not visible for downstream detectors.

These data have been compared with a full Geant 4 simulation of the setup, whose results are shown in figure 3 (left). Apart from the three peaks, the overall dependence of the spark probability with the beam energy is reproduced, and a fair agreement is quantitatively obtained with a Raether limit of $4 \times 10^{7}$. However, the simulation indicates that the spark probability does not depend on the charge of the pions. A similar simulation has been run with protons, and a narrow peak showed up around $300 \mathrm{MeV} / \mathrm{c}$, as illustrated on figure 3 (left). This peak originates from the stopping of protons in the detectors volume, as in figure 3 (right), which leads to the release of a very large number of primary electrons. This effect not only explains the absolute 

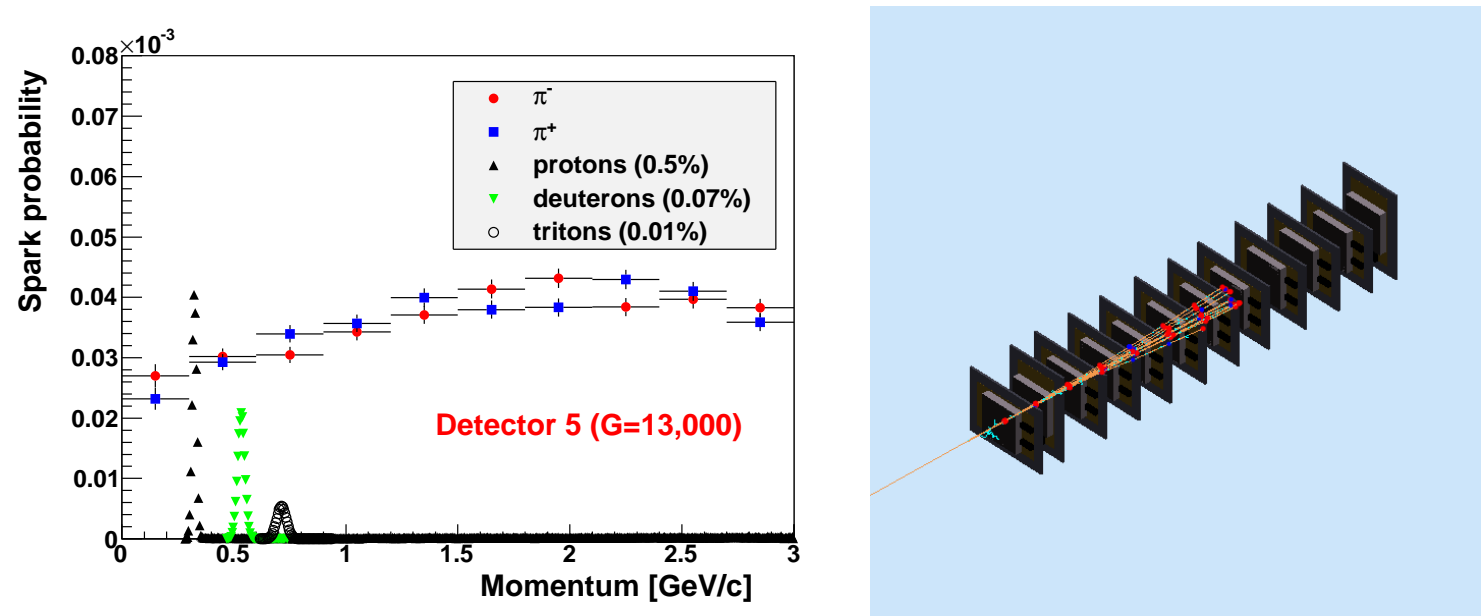

Figure 3. (Left) Simulated spark probability as a function of incident hadron momentum for pions, protons, deuterons and tritons, for the middle detector [10]. (Right) $300 \mathrm{MeV} / \mathrm{c}$ proton beam on the experimental PS setup, illustrating the stopping of protons.

position of the first peak in the data, but also its shift as a function of the detectors position. A higher energy is indeed needed for protons to reach and stop in the most downstream detectors. Last but not least, the correct amplitude of the peak could be obtained by assuming a $0.5 \%$ proton contamination in the $\pi^{+}$beam, which is compatible with observations from the scintillators $(<1 \%)$. The simulation has also been run with deuterons and tritons, and similar peaks indeed arised at 500 and $650 \mathrm{MeV} / \mathrm{c}$.

These two tests at high and low energies, combined with early measurements at $15 \mathrm{GeV} / \mathrm{c}$, confirmed the same Geant 4 based simulation can give a correct estimate of the spark probability over three orders of magnitude in beam momentum. The only free parameter is the Raether limit, which is found to be a few $10^{7}$ for all the measurements, and seems therefore intrinsic to the detectors. Besides, this value is close to the one generally assumed for the development of a spark, giving strong credit to the model.

\section{Sparks and magnetic field}

All the considerations above assumes that a spark is initiated by a constant number of electrons in the vicinity of the amplification gap. One should keep in mind, however, that the Raether limit was initially estimated from single avalanche processes. But in our case, a spark essentially originates from the stopping of a highly ionizing particle which leads to many, localized avalanches. It is therefore relatively intuitive that the charge density is a more relevant parameter than the total number of electrons. A simple way to emphasize the role of the charge density is to apply an external magnetic field which will modify the electrons drift inside the detector. The possible effect on the spark probability is likely to be different according to the direction of the magnetic field, i.e. perpendicular or parallel to the internal electric field. 


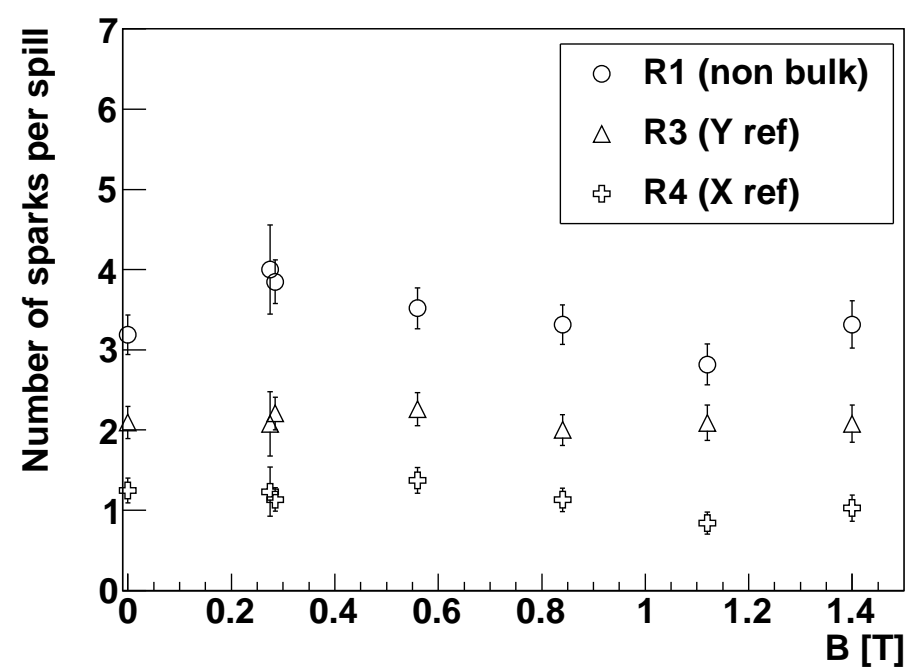

Figure 4. Number of sparks per spill for three detectors as a function of the transverse magnetic field. The Lorentz angle in the drift gap was estimated to be $20^{\circ}$ at $1.5 \mathrm{~T}$ [9].

\subsection{Case of a transverse magnetic field}

In the perpendicular configuration primary electrons drift along an axis with a Lorentz angle $\theta$ with respect to the electric field $\vec{E}[11]$ given by:

$$
\tan (\theta)=\frac{v \times B}{E},
$$

where $v$ is the electron drift velocity. Even in the case of a localized energy deposit, this angle will geometrically decrease the charge density. For a $20^{\circ}$ Lorentz angle, this reduction is of the order of $15 \%$.

The perpendicular configuration was investigated during the CERN/SPS beam test, where the detectors were located inside the Goliath magnet. This dipole delivers a tunable field up to $1.5 \mathrm{~T}$. The spark probability measured as a function of B is shown in figure 4. No B dependence has been observed with a Lorentz angle of $20^{\circ}$, though hints of a significant decrease have been seen in later measurements with a larger Lorentz angle.

\subsection{Case of a longitudinal magnetic field}

In the parallel case the electrons are focused around the magnetic field lines, resulting in a reduced transverse diffusion and a higher charge density. The study of this configuration took place in the Hall B of the Jefferson Laboratory in July 2010 [12]. A small Micromegas was inserted in the $5 \mathrm{~T}$ FROST solenoid, dowsntream of a $\mathrm{CH}_{2}$ target. The incident photon beam was obtained from the interaction of a primary $5.57 \mathrm{GeV}$ electron beam with a thin Pt radiator. The main characteristics of the setup are summarized in table 2 .

In a first set of measurements, the spark probability was recorded as a function of the gain, and agreed well with the Geant 4 simulation using a Raether limit of $2.5 \times 10^{7}$. In a second step, the gain of the detector was fixed and the magnetic field varied between 0 and $5 \mathrm{~T}$. An increase by a factor of 10 was obtained, as illustrated in figure 5. It was checked with the simulation that 
Table 2. JLab beam test specificities.

\begin{tabular}{|c|c|}
\hline Location & JLab/Hall B \\
\hline Beam momentum & 0 to $5.57 \mathrm{GeV}$ (Bremsstrahlung) \\
\hline Beam particles & photons \\
\hline Target & 19.3 mm thick $\mathrm{CH}_{2}$ \\
\hline Beam structure & continuous beam \\
\hline Max beam intensity & $2.5 \times 10^{9}$ photons $/ \mathrm{s}$ \\
\hline Number of detectors tested & 2 \\
\hline Detectors dimension & $11 \mathrm{~cm} \mathrm{diameter}$ \\
\hline Gas mixture & $\mathrm{Ar}+10 \% \mathrm{iC}_{4} \mathrm{H}_{10}$ \\
\hline Number of sparks recorded & 24,000 \\
\hline
\end{tabular}

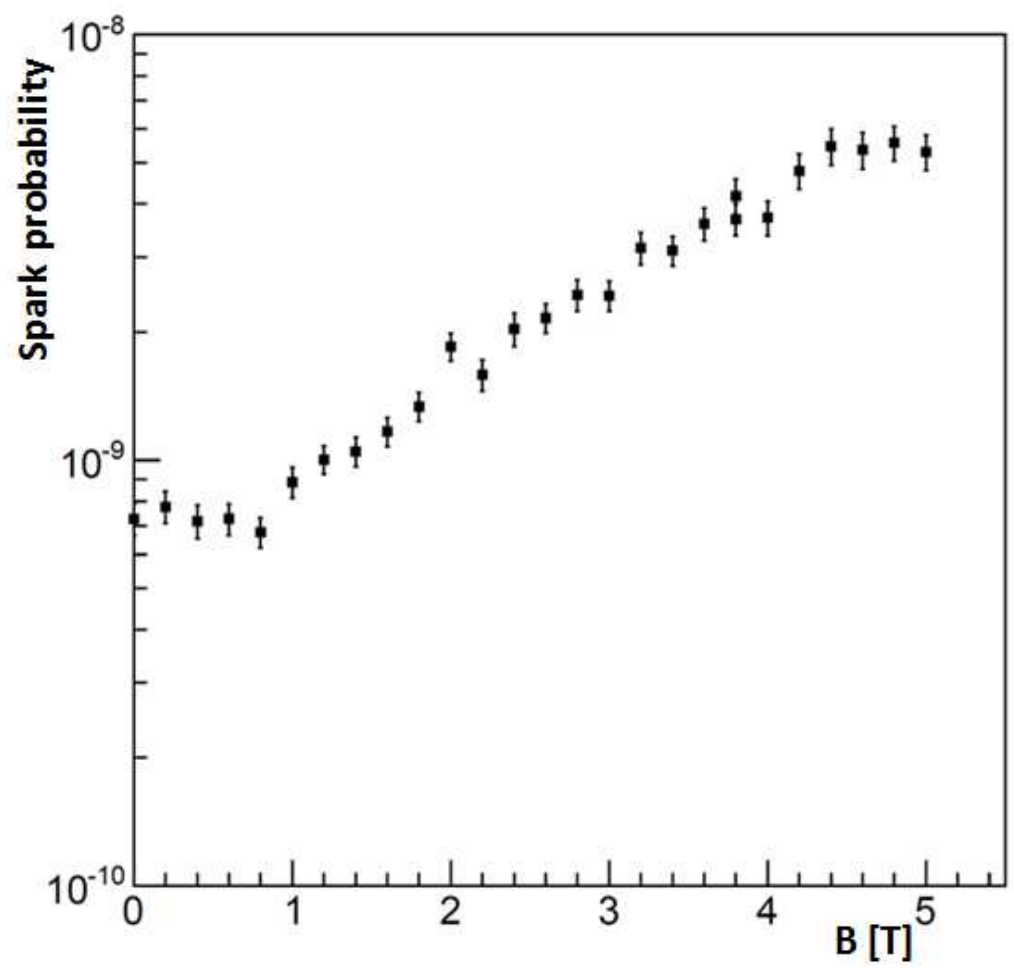

Figure 5. Spark probability per incident photon on the target as a function of a longitudinal magnetic field. The detector gain was approximately 1,000 [12].

the particle flux (especially hadrons) did not change significantly from 0 to $5 \mathrm{~T}$, proving that this increase can only be attributed to an enhancement of the charge density in the amplification gap. This effect was confirmed in a $1.5 \mathrm{~T}$ field using a ${ }^{241} \mathrm{Am}$ source, where the detector gain was shown to be independent on the magnetic field.

These measurements confirm unambiguously that the relevant parameter in spark development is indeed the charge density. The initial Geant4 simulation therefore needs to be upgraded to take 

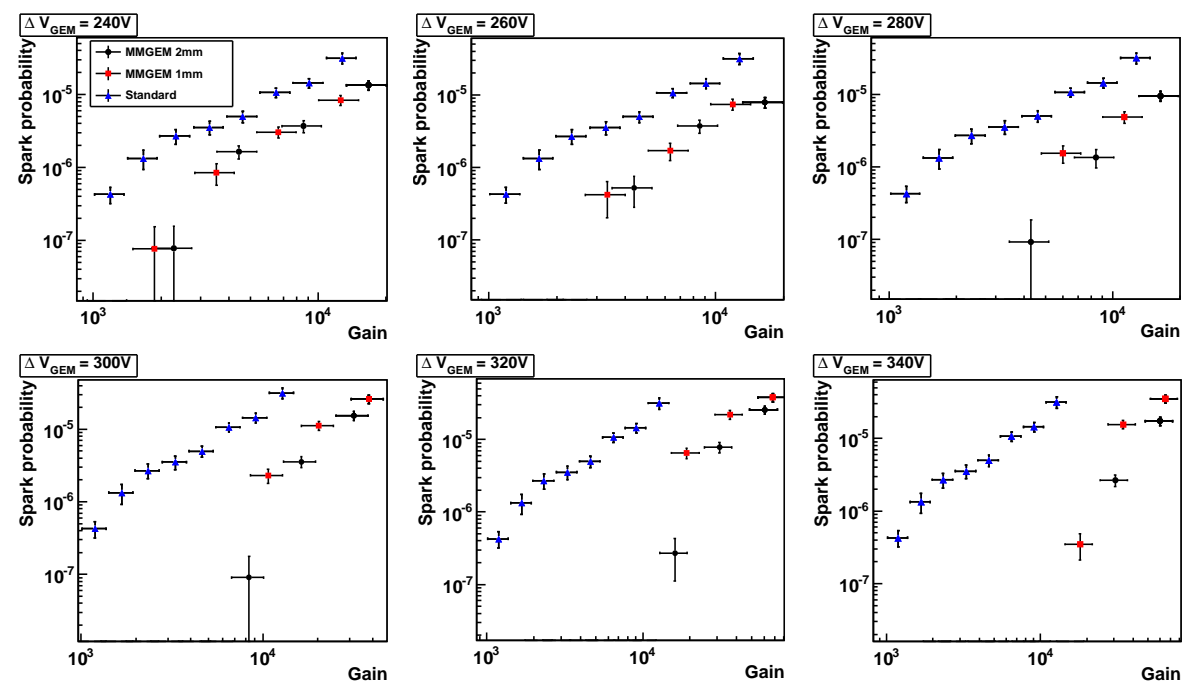

Figure 6. Spark probability as a function of the gain for MM-GEM detectors in a $3 \mathrm{GeV} / \mathrm{c}$ pion beam, and for different GEM gains. Data from a standard Micromegas are shown for comparison [10].

into account the transverse diffusion in the gas volume of the detector. This upgrade is described in the next section and illustrated in the case of a hybrid Micromegas-GEM detector.

\section{Effect of the GEM foil on the spark rate}

\subsection{Experimental observations}

It has been known for a long time that the addition of a GEM foil to a Micromegas detector significantly reduces the spark probability [8]. Precise measurements performed at CERN/SPS, PS, and JLab confirmed the reduction by a factor of 10 to 100, independently of the beam energy and experimental setup. To better understand the role played by the GEM in this reduction, a systematic study was performed during the CERN/PS beam test, where two Micromegas-GEM (MM-GEM) with 1 and $2 \mathrm{~mm}$ transfer gap ${ }^{1}$ were compared with a standard Micromegas. The resulting spark probabilities are shown in figure 6 as a function of the total gain and for different GEM high voltages. Several important observations can be made:

1. A significant reduction of the spark probability can be obtained with modest GEM gains (less than 10).

2. For moderate GEM gains the spark probability does not depend on the transfer gap size.

3. For larger GEM gains a larger transfer gap further reduces the spark probability.

4. The reduction of the spark probability seems to saturate at high GEM gains.

5. The slope of the spark probability as a function of the total gain increases at small gains for MM-GEM, in the large GEM gain domain. This tendency can actually be seen for the standard Micromegas at very small gain.

\footnotetext{
${ }^{1}$ The transfer gap is the distance between the micro-mesh and the GEM foil.
} 


\subsection{Critical surface charge density}

All the effects described above cannot be explained in a model where the spark is only initiated by the creation of a critical number of electrons. It should be mentioned, however, that the importance of the density was already partly taken into account in the original simulation, as the model considered large energy deposits occurring in a relatively small volume. The large energy deposits were then converted into a number of electrons $N$, and this lead to a discharge whenever the gain $G$ verified $N \times G \geq N_{R}$, where $N_{R}$ is of the order of a few $10^{7}$. However, this simulation did not distinguish from energy deposits appearing at different heights in the detector volume. Because of the transverse diffusion of the primary electrons, a given energy deposit is more likely to generate a discharge if occurring close to the micro-mesh.

In the new simulation, a pointlike energy deposit $E_{\text {dep }}$ is converted into a surface charge density $d_{S}$ by estimating the mean area of the electron cloud at the readout strip level. The transverse extension of the cloud being proportional to the square root of the distance $z$ to the readout strips (usual diffusion model), one can write:

$$
d_{S}=\frac{4 \times G \times E_{\mathrm{dep}}}{\pi w_{i}(a(E, B) \times \sqrt{z})^{2}},
$$

where $w_{i}$ is the ionization potential of the gas. The parameter $a(E, B)$ is closely related to the transverse diffusion of the gas, and can be estimated through Garfield simulation for any electric and magnetic fields. In practice, a systematic transverse diffusion of $100 \mu \mathrm{m}$ was added to the term in $a \times \sqrt{z}$ to account for the additional spread coming from the avalanche in the amplification gap.

In the case of a MM-GEM detector, the gain appearing in the above equation is the total gain if the energy deposit occurs in the conversion region, and only the Micromegas one if it occurs in the transfer gap. The sparking condition now reads:

$$
d_{S} \geq d_{S}^{\lim },
$$

where $d_{S}^{\lim }$ is a critical surface charge density.

\subsection{Comparison with data}

The resulting discharge probabilities obtained with either the Raether or the density limit are shown in figure 7. The density limit exhibits a very similar behaviour with the gain, though with a slightly smaller slope. The adjustment gives:

$$
d_{S}^{\lim }=2 \times 10^{9} / \mathrm{mm}^{2}
$$

It simply means that the data reproduced by the Raether condition can alternatively be described in terms of a critical charge density.

The same simulation was applied to two MM-GEM detectors with 1 and $2 \mathrm{~mm}$ transfer gaps respectively. The figure 8 (left) illustrates the effects of both the GEM gain and the transfer gap on the spark probability. These effects are in qualitative agreement with observations made above, namely: 


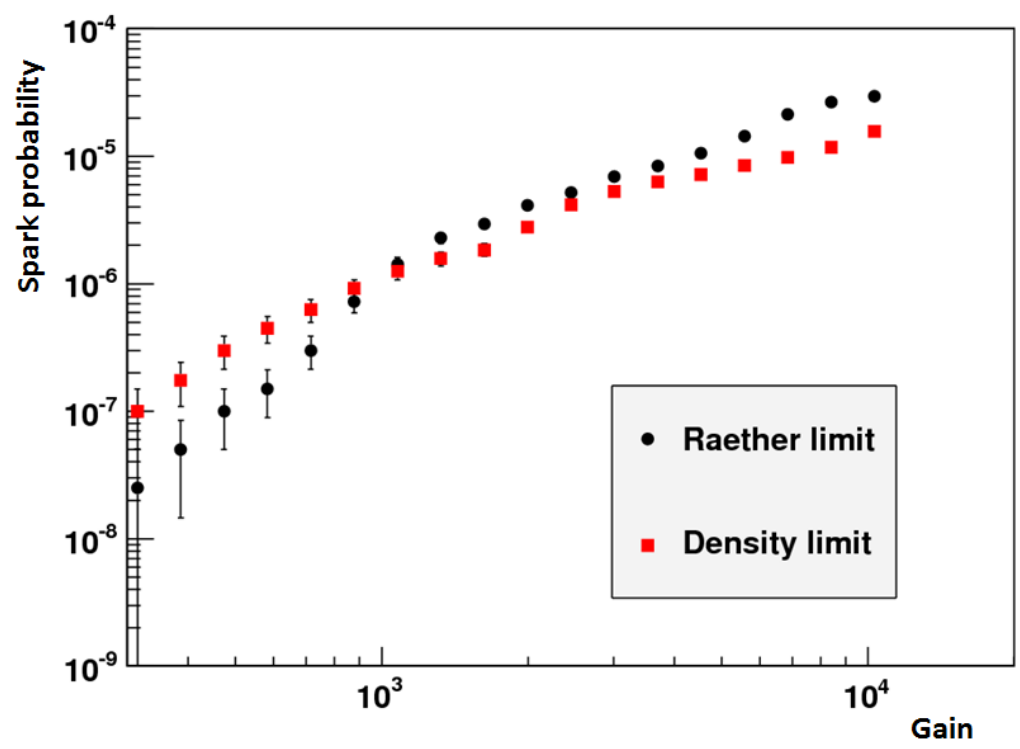

Figure 7. Discharge probability obtained with a Geant 4 simulation of $3 \mathrm{GeV} / \mathrm{c} \pi^{-}$, using either a Raether or a density limit.

- For small GEM gains at a fixed total gain, most of the sparks originate from energy deposits occurring in the transfer gap where only the micro-mesh gain plays a role. These sparks are suppressed when transferring a small part of the gain from the micro-mesh to the GEM (1). In this regime, the size of the transfer gap is of no importance (2).

- For large GEM gains, all the sparks initiated by energy deposits in the transfer gap have been suppressed. The observed sparks now come from energy deposits in the drift gap. These energy deposits being amplified by the total gain, a further transfer of the gain from the micro-mesh to the GEM does not further suppress the sparks (4). In this case, a higher transfer gap enhances the transverse diffusion and thus lowers the charge density (3).

- For large GEM gains and very small micro-mesh gains, the energy deposit required to initiate a spark becomes higher than a deposit corresponding to the stopping of a highly ionizing particle. In this regime the slope of the spark probability as a function of the total gain is strongly enhanced (5).

A quantitative comparison between the simulation and the data is shown in figure 8 (right) for an intermediate GEM gain. Using the same critical charge density as above, a fair agreement is obtained simultaneously for the standard Micromegas and the two MM-GEM.

\section{Conclusion}

An extensive study of sparks in MPGD has been performed in various environments. Precise spark probabilities are now available on a wide range of beam energy, and all the measurements confirm the validity of the Geant 4 based simulation. Dedicated beam studies in magnetic fields clearly showed that the charge density is a more relevant parameter than the usual Raether limit 

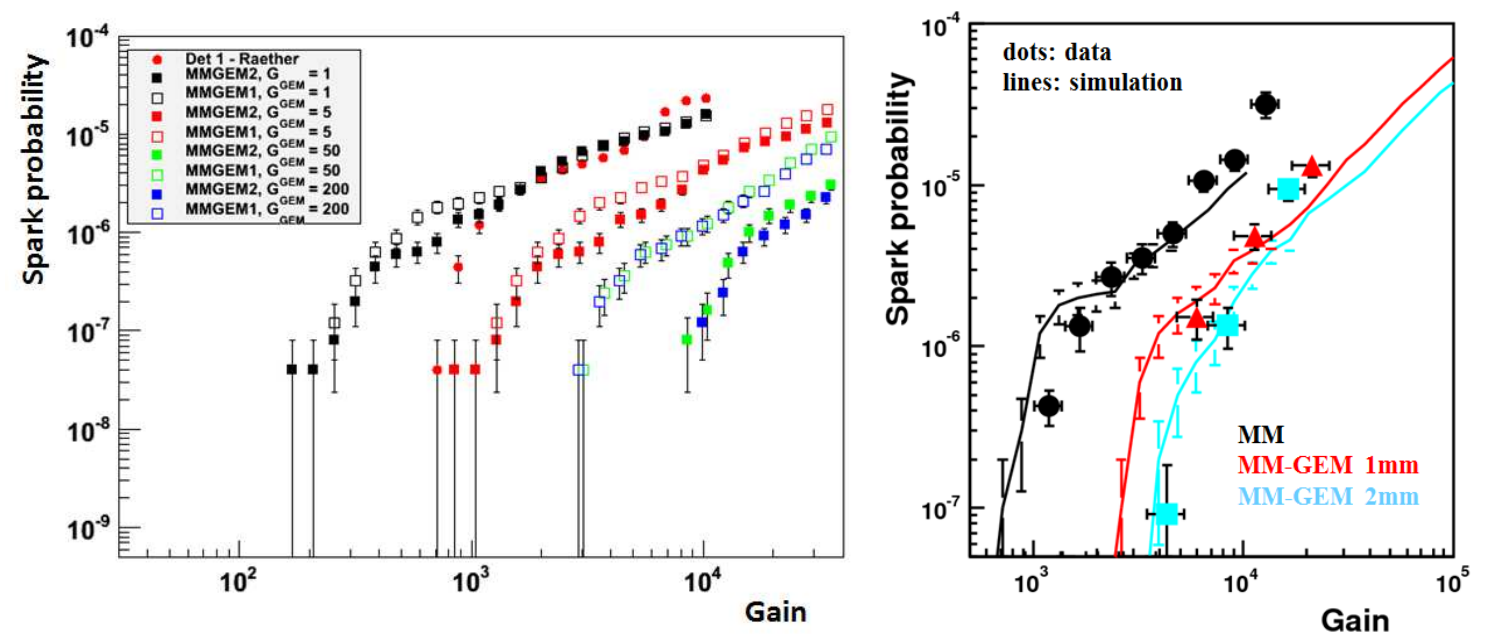

Figure 8. (Left) Simulated spark probability in the 1 and $2 \mathrm{~mm}$ MM-GEM as a function of the total gain, for different GEM gains. (Right) Comparison of the spark probability from the data (points) and from the simulation (lines) for the Micromegas and the two MM-GEM (with a GEM gain of 30).

to describe the spark development. The simulation has been modified accordingly to take into account the transverse diffusion of primary electrons in the gas, and a critical charge density of $2 \times 10^{9}$ electrons $/ \mathrm{mm}^{2}$ has been derived. The upgraded simulation has been applied successfully to hybrid Micromegas-GEM detectors, leading to a quantitative understanding of the spark reduction with GEM foils. The model therefore provides a reliable tool to predict and minimize the spark rate for a given setup, and could certainly be extended to other MPGDs.

\section{References}

[1] H. Raether, Die Entwicklung der Elektronenlawine in den Funkenkanal, Z. Phys. 112 (1939) 464.

[2] GeAnt4 collaboration, S. Agostinelli et al., Geant4 - a simulation toolkit, Nucl. Instrum. Meth. A 506 (2003) 250.

[3] Y. Giomataris, P. Rebourgeard, J.P. Robert and G. Charpak, MICROMEGAS: a high-granularity position-sensitive gaseous detector for high particle-flux environments, Nucl. Instrum. Meth. A 376 (1996) 29.

[4] S. Procureur et al., A Geant4-based study on the origin of the sparks in a Micromegas detector and estimate of the spark probability with hadron beams, Nucl. Instrum. Meth. A 621 (2010) 177.

[5] D. Thers et al., Micromegas as a large microstrip detector for the COMPASS experiment, Nucl. Instrum. Meth. A 469 (2001) 133.

[6] Y. Giomataris et al., Micromegas in a bulk, Nucl. Instrum. Meth. A 560 (2006) 405 [physics/0501003].

[7] F. Sauli, GEM: a new concept for electron amplification in gas detectors, Nucl. Instrum. Meth. A 386 (1997) 531.

[8] S. Kane et al., A study of Micromegas with preamplification with single GEM, in Proceedings of the 7th International Conference on Advanced Technology and Particle Physics, Como Italy (2001). 
[9] S. Procureur et al., Discharge studies in micromegas detectors in a $150 \mathrm{GeV/c}$ pion beam, Nucl. Instrum. Meth. A 659 (2011) 91.

[10] G. Charles et al., Discharge studies in Micromegas detectors in low energy hadron beams, Nucl. Instrum. Meth. A 648 (2011) 174.

[11] P. Konczykowski et al., Measurements of the Lorentz angle with a Micromegas detector in high transverse magnetic fields, Nucl. Instrum. Meth. A 612 (2010) 274.

[12] B. Moreno et al., Discharge rate measurements for Micromegas detectors in the presence of a longitudinal magnetic field, Nucl. Instrum. Meth. A 654 (2011) 135. 